\title{
Efeitos de um evento de friagem nas condições meteorológicas na Amazônia: um estudo de caso.
}

\author{
Paulo Jorge de OLIVEIRA ${ }^{1}$, Edson José Paulino da ROCHA², Gilberto FISCH ${ }^{3}$, Bart KRUIJT ${ }^{4}$, João Batista \\ Miranda RIBEIRO ${ }^{2}$
}

\section{RESUMO}

A influência do fenômeno da friagem nas variáveis meteorológicas e nos fluxos de energia e $\mathrm{CO}_{2}$, foi realizada numa área de floresta próxima à região de Ji-Paraná, Rondônia, durante o mês de junho de 2001. A friagem ocasionou uma diminuição de $35 \%$ no valor da temperatura do ar. Notou-se uma redução de $75 \mathrm{~W} \cdot \mathrm{m}^{-2}$, na radiação solar incidente, associada à nebulosidade presente em decorrência da penetração da massa de ar fria. Conseqüentemente, tanto o fluxo de calor sensível (H) quanto o de calor latente (LE), apresentaram uma diminuição no seu valor médio diário, apresentando uma diferença em relação aos dias normais de 8 e 34\%, respectivamente. A concentração de $\mathrm{CO}_{2}$ permaneceu constante, sem apresentar o aumento comum durante a noite, devido à condição de vento forte típica da friagem. Em situação normal o fluxo médio durante o dia de $\mathrm{CO}_{2}\left(-2,44 \mathrm{mmol} \mathrm{m}^{-2} \mathrm{~s}^{-1}\right)$ foi menor que durante os dias de friagem $\left(-5,78 \mathrm{mmol} \mathrm{m}^{-2} \mathrm{~s}^{-1}\right)$; enquanto que os fluxos médios noturnos foram $+1,77 \mathrm{mmol} \mathrm{m}^{-2} \mathrm{~s}^{-1} \mathrm{e}+2,83 \mathrm{mmol} \mathrm{m}^{-2} \mathrm{~s}^{-1}$ durante situações de dias normais e de friagem, respectivamente.

\section{PALAVRAS-CHAVE}

Amazônia, Friagem, Clima, Fluxo de Carbono

\section{Meteorological effects of a cold spell event over Amazonia: a case study.}

\begin{abstract}
Observations of the influence of a Cold Spell phenomena on meteorological variables and on energy and $\mathrm{CO}_{2}$ fluxes were made in a forest site near Ji-Paraná, Rondonia, during June 2001. Friagem caused a diminution of 35\% at air temperature. A reduction of $75 \mathrm{W.m}^{-2}$ from normal days $\left(200 \mathrm{W.m} \mathrm{W}^{-2}\right)$ to cold days (125 W.m. $\left.{ }^{-2}\right)$ in the mean incoming solar radiation was also observed. During the Cold Spell days, both sensible heat (H) and latent heat fluxes (LE) showed a decrease in their mean daily value, showing a difference from normal days of 8 e $34 \%$, respectively. The $\mathrm{CO}_{2}$ concentration remained constant, without increase during the night, due to the windy condition of cold days. During normal days the mean diurnal $\mathrm{CO}_{2}$ flux $\left(-2,44 \mathrm{mmol} \mathrm{m}^{-2} \mathrm{~s}^{-1}\right)$ was lesser than that one at phenomena days $\left(-5,78 \mathrm{mmol} \mathrm{m}^{-2} \mathrm{~s}^{-1}\right)$, while the mean nocturnal fluxes were $+1,77 \mathrm{mmol} \mathrm{m} \mathrm{m}^{-2} \mathrm{~s}^{-1} e+2,83 \mathrm{mmol} \mathrm{m} \mathrm{m}^{-2}$ during normal and cold days, respectively.
\end{abstract}

\section{KEY WORDS}

Amazonian Rain Forest, Friagem, Climate, Carbon budget

\section{INTRODUÇÃO}

A Região Amazônica é importante por apresentar uma grande diversidade com relação à fauna e flora, e também por possuir um clima tropical úmido. Sua localização geográfica faz com que durante todo o ano haja uma grande quantidade de energia disponível no local, gerando desta forma um forte interesse na compreensão das formas com que esta energia é usada na interação solo-florestaatmosfera. Acredita-se também que, devido a sua extensão, a Floresta Amazônica tenha um importante papel no balanço global, de calor, umidade e de carbono (Fitzjarrald \& Moore, 1990). Entretanto, apenas nas últimas décadas (particularmente após a década d 1980), esta região tem sido exaustivamente estudada, através de grandes experimentos meteorológicos (Fisch, et al., 1998).

\footnotetext{
${ }^{1}$ Universidade Federal Rural da Amazônia (UFRA), Belém, CEP 66077-530 00, PA, Brasil, pj@ufra.edu.br

${ }^{2}$ Universidade Federal do Pará (UFPa), Belém, CEP 66000-000, PA, Brasil

${ }^{3}$ Centro Técnico Aeroespacial (CTA/IAE-ACA), São José dos Campos, CEP 12228-904, SP, Brasil

${ }^{4}$ ALTERRA, Wageningen, $6700 \mathrm{AA}$, The Netherlands
} 


\section{ACTA \\ AMAZONICA}

EFEITOS DE UM EVENTO DE FRIAGEM NAS CONDIÇÕES

METEOROLÓGICAS NA AMAZÔNIA: UM ESTUDO DE CASO
Em Rondônia, local do presente estudo, nota-se uma forte sazonalidade na distribuição da precipitação, com o período chuvoso (novembro-abril) apresentando totais mensais acima de $200 \mathrm{~mm}$.mês ${ }^{-1}$. A estação menos chuvosa estende-se de maio a outubro, com um pequeno período de poucas chuvas entre junho-agosto, com total mensal inferior a $20 \mathrm{~mm}$.mês $\mathrm{s}^{-1}$. A temperatura do ar, para o mesmo período, mostra também uma sazonalidade, sendo Outubro o mês mais quente, com temperatura média de $25,6{ }^{\circ} \mathrm{C}$. O mês mais frio é Julho, com temperatura média próxima a $22,7^{\circ} \mathrm{C}$ (Culf et al., 1996, Von Randow et al., 2004).

Durante o inverno, a região de estudo sofre forte influência de frentes frias que atingem o sul da Amazônia (Fisch, 1995). Este fenômeno conhecido localmente como "Friagem", ocasiona uma brusca alteração nas condições meteorológicas, causando uma diminuição da temperatura e umidade do ar e modificando as características ambientais. Trabalhos anteriores descreveram friagem como sendo uma forte entrada de ar frio vindo do sul o qual penetra dentro dos trópicos e afeta a Amazônia (Serra \& Rastibona, 1942).

Outros estudos mais recentes (Fisch, 1995; Culf et al., 1996; Grace et al., 1996; Marengo et al., 1997; Oliveira et al., 2001 ; Fisch et al., 2004) descrevem as características deste fenômeno e sua influência na Amazônia, dando uma idéia das mudanças ocorridas nas condições de tempo da região. Segundo Fisch et al. (2004), em julho de 1993, na mesma região de estudo, a penetração de uma friagem de intensidade média ocasionou um valor de $15,1^{\circ} \mathrm{C}$ na temperatura mínima da superfície. Marengo et al. (1997) observaram que o tempo para que as variáveis meteorológicas voltem às condições normais, durante um evento de friagem, varia em média, entre 5 e 6 dias. Oliveira et al. (2001), verificaram que, devido ao aumento da intensidade do vento durante eventos de friagem, os fluxos noturnos de $\mathrm{CO}_{2}$ são superiores em relação a condições normais.

Este trabalho apresenta um estudo de caso do fenômeno de friagem em uma área de floresta em Rondônia ocorrido em junho de 2001, descrevendo o seu efeito não apenas nas condições meteorológicas bem como nos fluxos de água, energia e carbono.

\section{MÉTODOS}

\section{Localização}

O estudo foi realizado numa área de floresta no estado de Rondônia (Fig. 1), localizada cerca de $100 \mathrm{~km}$ do município de Ji-Paraná na Reserva Biológica do Jarú $\left(10^{\circ}\right.$ $05^{\prime} \mathrm{S}$ e $61^{\circ} 55^{\prime} \mathrm{W}, 120 \mathrm{~m}$ ), que possui 268.150 ha de floresta tropical intacta, mantida pelo Instituto Brasileiro do Meio Ambiente e dos Recursos Naturais Renováveis (IBAMA). Situa-se em uma região de floresta do tipo "terra firme", com árvores atingindo 33 metros de altura em média. Na Reserva Jarú, do setor noroeste para sul-sudeste, o qual representa a direção predominante do vento (Oliveira e Fisch, 2001), a área de influência da vegetação ("fetch") é representada por dezenas de quilômetros de floresta intacta. No setor restante esta área é menor, da ordem de $1 \mathrm{~km}$. O Rio Ji-Paraná fica em torno de $300 \mathrm{~m}$ a oeste da torre e forma o limite oeste da Reserva Jarú (Culf et al., 1996).

Oliveira e Fitzjarrald (1993) observaram a presença de uma circulação de brisa de rio próximo à cidade de Manaus, a qual era uma particularidade do local devido à presença dos rios Negro e Solimões. Embora a região de estudo fique próxima ao rio Ji-Paraná, não existem evidências deste tipo de influência, devido às pequenas dimensões do rio (Oliveira e Fisch, 2001).

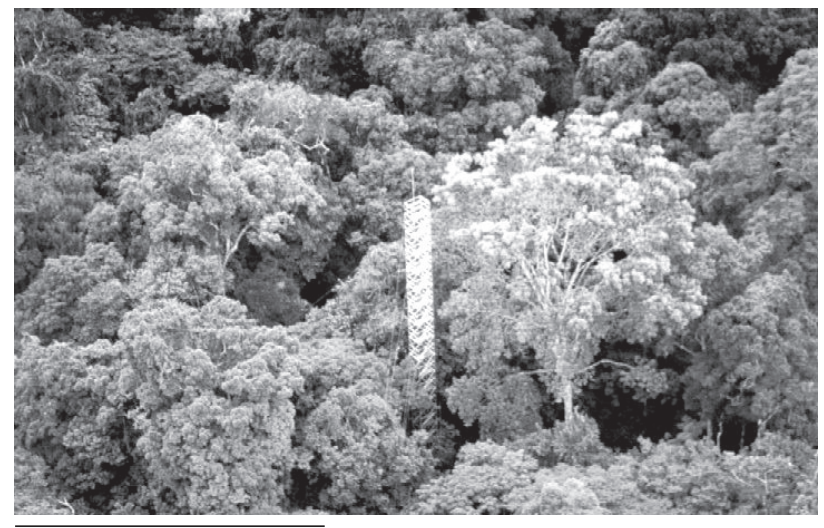

Figura 1 - Localização geográfica do sítio na região de Jí-Paraná-RO.

\section{Dados}

Foram utilizados nesse estudo dados micrometeorológicos e de fluxos de energia, vapor d'água e $\mathrm{CO}_{2}$ provenientes do Projeto Científico LBA (Kruijt et al., 2004, Von Randow et al., 2004). Os dados meteorológicos utilizados foram obtidos através de uma estação meteorológica automática (CR10X, Campbell Scientific, UK) fornecendo informações médias a cada 30 minutos e os fluxos de superfície foram medidos por um sistema de correlação de vórtices turbulentos (eddy covariance) do tipo "closed path" constituído por um anemômetro sônico 3D (Solent R-3, Gill instruments, Lymington, UK) e um analisador de gás infravermelho LiCor 6262 (LICOR, Lincoln, Nebraska, USA) (Moncrieff et al., 1997), o qual faz medidas instantâneas em alta freqüência $(20 \mathrm{~Hz})$ e fornece dados médios de 30 em 30 minutos.

Foram usados dados coletados por estes sistemas no período em que há maior ocorrência da friagem na região, que em junho de 2001 (dias Juliano 160 a 190) se estenderam por 4 dias (dias Juliano 169, 170, 171 e 172). Obtiveram-se valores médios das variáveis estudadas, e definiu-se como condições de dia de friagem a média dos 4 dias do evento. Como situação de dia normal utilizou-se o valor médio do restante dos dias. 


\section{ACTA AMAZONICA}

\section{RESULTADOS E DISCUSSÃO}

\section{Condições meteorológicas}

As condições meteorológicas observadas durante a passagem do evento de friagem são apresentadas na Figura 2 e na Tabela 1. Estes dados mostram o efeito que a friagem provoca: redução da temperatura do ar e da radiação solar incidente e diminuição da umidade do ar.

a)Temperatura do ar (Tar)

A friagem atingiu a região em torno das 12 horas Local (HL) do dia 168 (17 de junho) e foi caracterizada por uma brusca redução da temperatura média do ar, de $29^{\circ} \mathrm{C}$ para $14,3^{\circ} \mathrm{C}$, observado às $09 \mathrm{~h}$ do dia 169 (18 de junho), uma redução de quase $15^{\circ} \mathrm{C} \mathrm{em}$ menos de $24 \mathrm{~h}$ (Fig. 2). O menor valor de temperatura mínima do ar foi encontrado no dia 170 (19 de junho) próximo das $8 \mathrm{HL}$, com valor de $12,0^{\circ} \mathrm{C}$. Após este dia, a temperatura começou a aumentar gradativamente, alcançando $28,6^{\circ} \mathrm{C}$ às $17 \mathrm{~h}$ do dia 174 (23 de junho), cerca de 6 dias após a entrada da friagem. De acordo com Marengo et al. (1997), a amplitude das alterações causadas pela penetração do sistema indica a intensidade do fenômeno. Os mesmos autores observaram que o tempo para que as variáveis meteorológicas voltem

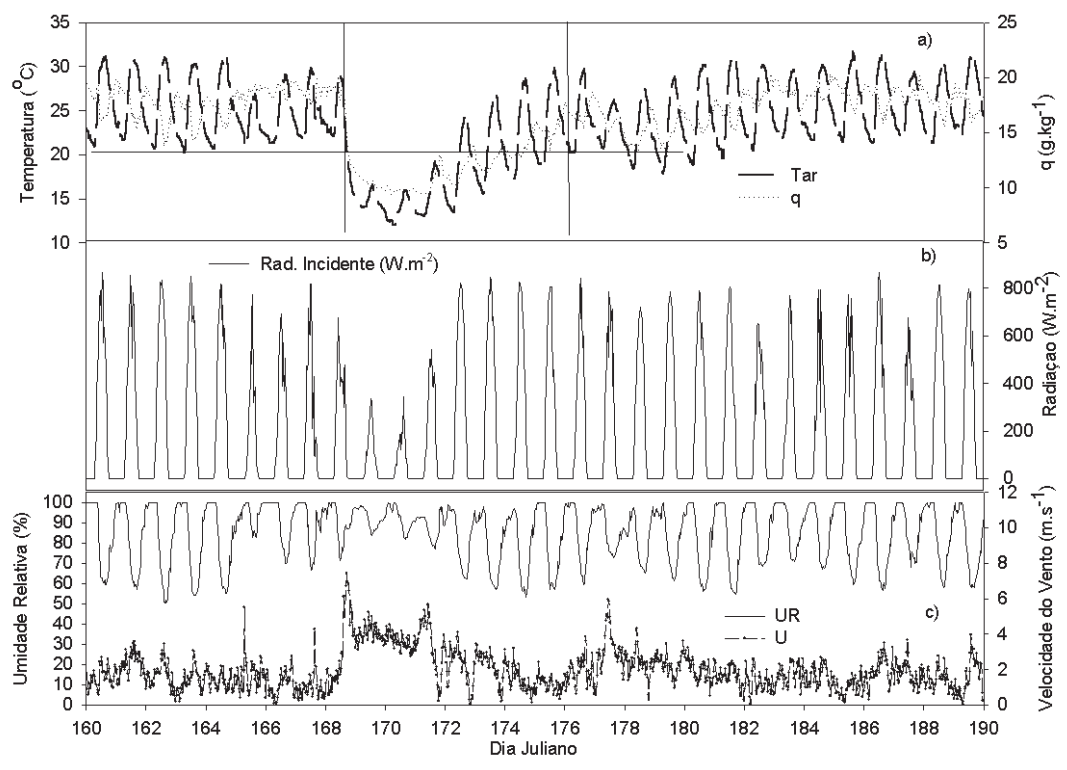

Figura 2 - Série Temporal das variáveis meteorológicas (Tar, q, Rad Inc, UR e U), durante o mês de Junho de 2001.

Tabela 1 - Média das Variáveis Meteorológicas para dias de Friagem e Normais.

\begin{tabular}{lcccccc}
\hline \hline & $\operatorname{Tar}\left({ }^{\circ} \mathrm{C}\right)$ & $\mathrm{Tmax}\left({ }^{\circ} \mathrm{C}\right)$ & $\mathrm{Tmin}\left({ }^{\circ} \mathrm{C}\right)$ & $\mathrm{U}\left(\mathrm{m}^{-} \mathrm{s}^{-1}\right)$ & $\mathrm{q}\left(\mathrm{g} \cdot \mathrm{kg}^{-1}\right)$ & $\mathrm{R} \cdot \mathrm{in}\left(\mathrm{W} \cdot \mathrm{m}^{-2}\right)$ \\
\hline Dia normal & $24,6( \pm 0,3)$ & $29,7( \pm 0,3)$ & $20,1( \pm 0,3)$ & $1,7( \pm 0,1)$ & $16,1( \pm 0,3)$ & $200,6( \pm 59,4)$ \\
Dia de friagem & $15,6( \pm 1,0)$ & $19,0( \pm 1,9)$ & $13,0( \pm 0,4)$ & $3,4( \pm 0,1)$ & $9,7( \pm 0,4)$ & $125,4( \pm 41,0)$ \\
Diferença & $-9,0$ & $-10,7$ & $-7,1$ & 1,7 & $-6,4$ & $-75,2$ \\
\hline$\%)$ & -36 & -36 & -35 & 97 & -40 & -38 \\
\hline \hline
\end{tabular}

às condições normais varia em média, entre 5 e 6 dias. Fisch (1995), fez uma observação de um evento de friagem, classificado como moderado, ocorrido em julho de 1993, e notou que para a mesma região de estudo, a temperatura mínima do ar atingiu $15,1^{\circ} \mathrm{C}$, uma diminuição de $6,6^{\circ} \mathrm{C}$ em relação ao dia anterior. Curiosamente, durante um evento de friagem ocorrido na mesma região em 1994, a temperatura do ar reduziu-se de $22,5^{\circ} \mathrm{C}$ no dia 25 de junho para $11,3{ }^{\circ} \mathrm{C}$ no dia 26 (Marengo et al.,1997).

A Tabela 1 apresenta valores médios diários das variáveis meteorológicas em dias normais e de friagem, onde se nota que as temperaturas do ar média, mínima e máxima, apresentaram uma diminuição em torno de 35\% durante os dias de friagem. Em dias normais, a temperatura mínima média encontrada foi de $20,1^{\circ} \mathrm{C}$; com a influência do sistema, este valor diminuiu para $13^{\circ} \mathrm{C}$, uma diferença média cerca de $7^{\circ} \mathrm{C}$.

b)Umidade específica do ar (q)

A umidade específica do ar (Fig. 2), apresentou a mesma característica da temperatura do ar, com valor médio de 20,1 g. $\mathrm{kg}^{-1}$ antes da entrada da friagem (10 HL do dia 168), diminuindo $49,5 \%$ no dia 169 às $12 \mathrm{HL}$ (com um valor de $10,2 \mathrm{~g} \cdot \mathrm{kg}^{-1}$ ). O valor mínimo de $q$ foi encontrado um dia após o mínimo valor da temperatura, atingindo 9,4 g. $\mathrm{kg}^{-1}$ às $10: 30$ HL do dia 171. De acordo com Fisch (1995), a friagem de 1993 também reduziu a umidade específica em cerca de 5,0 g. $\mathrm{kg}^{-1}$ na superfície e em torno de $3,0 \mathrm{~g} \cdot \mathrm{kg}^{-1}$ dentro da camada limite convectiva até $1,2 \mathrm{~km}$ de altura. Durante dias normais, o valor médio da umidade específica média chegou a 16,1 g. $\mathrm{kg}^{-1}$; o efeito da friagem causou uma redução média de 6,5 g.kg ${ }^{1}$, uma diferença de cerca de $40 \%$ de uma condição para outra (Tab. 1)

c)Velocidade do vento (U)

Segundo Marengo et al. (1997), a entrada da friagem no dia 26 de junho de 1994 na Amazônia ocasionou um aumento na velocidade do vento da ordem de duas vezes a intensidade do valor do dia anterior, atingindo $8,0 \mathrm{~m} . \mathrm{s}^{-1}$, com direção predominante de sul. A Figura 2 mostra que durante a entrada e permanência do sistema, houve um aumento na intensidade do vento, podendo-se observar valores de até 6,0 7,0 m.s. ${ }^{-1}$ já no início do período de friagem (17 HL, dia 168), enquanto nos dias anteriores o valor máximo encontrado foi de 4,3 m.s. ${ }^{-1}$. Em média, notou-se um aumento de $97 \%$ na intensidade do vento devido à influência da friagem, com valores médios diários de $1,7 \mathrm{e} \mathrm{3,4}$ m.s. ${ }^{-1}$, durante dias normais e de friagem, respectivamente (Tab. 1). 


\section{ACTA AMAZONICA}

EFEITOS DE UM EVENTO DE FRIAGEM NAS CONDIÇÕES METEOROLÓGICAS NA AMAZÔNIA: UM ESTUDO DE CASO

\section{d)Radiação Solar Incidente (R.in)}

A radiação solar incidente na superfície depende diretamente da quantidade de nuvens, o qual aumenta consideravelmente com a penetração de sistemas frontais (Fig. 3). Neste estudo de caso, notou-se uma diminuição gradual na radiação incidente ao longo do período afetado pela friagem. Do mesmo modo que a temperatura, a radiação apresentou um decréscimo a partir do dia 168 (17 de junho) as 10:30 HL, chegando a um valor máximo de $679{\mathrm{~W} . \mathrm{m}^{-2}}^{2}$ reduzindo-se em seguida para um valor de $388 \mathrm{~W} \cdot \mathrm{m}^{-2}$ às 12 HL, indicando o início da influência da friagem. No dia anterior à friagem (167), o valor médio para este horário (12 HL) foi de 844 W.m.-2 , uma diminuição de 54\%. No dia 169 (18 de junho), a máxima radiação incidente foi menor ainda, com valor de 336 W.m ${ }^{-2}$ às 12:30 HL. Na Figura 2, nota-se que a radiação incidente apresenta uma tendência a retornar ao seu ciclo diurno normal no dia 170 (19 de junho), com um valor de $187 \mathrm{~W} . \mathrm{m}^{-2}$ às 12:50 HL, aumentando em seguida para $345 \mathrm{~W} . \mathrm{m}^{-2}$ às 14:30 HL. O valor médio de máxima radiação incidente foi obtido apenas no dia 172, cerca de 5 dias após o início da friagem, com valor de $824 \mathrm{~W} . \mathrm{m}^{-2}$ às $12: 30 \mathrm{~h}$. Segundo Fisch (1995), a média diária da radiação solar incidente caiu de 197,8 W.m ${ }^{-2}$ (6 de julho de 1993) para um valor de 82,1 W. $\mathrm{m}^{-2}$ em 7 de julho, alcançando 199 W.m ${ }^{-2}$ no próximo dia.

A radiação solar incidente média diária sofreu uma redução devido ao aumento da nebulosidade proveniente do sistema frontal (Fig. 3). A diferença média foi de 37\%, uma redução de $75 \mathrm{~W} . \mathrm{m}^{-2}$, de dias normais $\left(200 \mathrm{~W} \cdot \mathrm{m}^{-2}\right)$ para dias de friagem (125 W.m.2) (Tab. 1).


Figura 3(a-g) - Cobertura de Nuvens durante um evento de Friagem (dias 167-172). (Fonte: GOES-8 IR, www.cptec.inpe.br) anteriormente.

\section{e)Condições Sinóticas}

A formação de nuvens associada à entrada da friagem pode ser visualizada na Figura 3a-g, a qual apresenta uma seqüência temporal da evolução da nebulosidade durante o evento às 10:45 HL. Nota-se que no dia 16 de junho (Dia Juliano 167), a região de estudo não apresentava praticamente nenhuma cobertura de nuvens (Fig. 3a). No dia 168 (17 de junho) a nebulosidade já cobria totalmente o estado de Rondônia (Fig. 3b), exatamente quando se observa o início da entrada da friagem, perceptível pela declinação da temperatura do ar apresentada na figura 2 . A partir deste dia percebe-se que a cobertura de nuvens é total sobre a região, permanecendo assim durante os dias seguintes: dias juliano 169, 170 e 171 (Fig. 3c-e). Este aumento na nebulosidade foi o responsável pela redução de $37 \%$ na radiação incidente, conforme visto

No dia 172 (21 de junho), a nebulosidade começa a diminuir, marcando o término da influência do sistema na região (Fig. 3f). Somente no dia 173 (22 de junho) é que a nebulosidade dissipa-se, associada ao movimento de subsidência presente após a passagem do sistema.

\section{Balanço de Energia}

Analisando a Figura 4, pode-se observar uma forte diminuição nas componentes do balanço de energia, devido à redução na radiação solar incidente $\mathrm{e}$, conseqüentemente, no saldo de radiação $(\mathrm{Rn})$. A Figura 5 representa o ciclo diurno do balanço de energia médio durante dias de friagem (a) e dias normais (b). Nota-se que durante dias normais, o valor máximo de Rn foi de 533 W. $\mathrm{m}^{-2}$, havendo uma redução de $200 \mathrm{~W} \cdot \mathrm{m}^{-2}$ em relação ao mesmo valor de Rn em dias de friagem (valor médio de $333 \mathrm{~W} \cdot \mathrm{m}^{-2}$ ).

Durante dias de friagem, tanto o fluxo de calor sensível $(\mathrm{H})$ quanto o fluxo de calor latente (LE), apresentaram uma diminuição no seu valor máximo diário, sendo esta redução mais intensa no caso de LE (Fig. 5). Em dias normais encontrouse valor máximo diários de 341 e 120 W. $\mathrm{m}^{-2}$ para LE e $\mathrm{H}$, respectivamente, enquanto que em dias de friagem estes valores máximos atingiram apenas 146 e 103 W. $\mathrm{m}^{-2}$, respectivamente. Nota-se também que apesar de haver uma forte queda na temperatura do ar, a maior redução foi observada na componente LE. Em três dias consecutivos de influência de friagem, Fisch (1995) encontrou valores médios diários de $\mathrm{H}$ de $20,8 \mathrm{~W} \cdot \mathrm{m}^{-2}$ no 


\section{ACTA AMAZONICA}

dia 6/7/1993; 8,1 W.m ${ }^{-2}$ no dia 7/7/1993 e 40,5 W.m ${ }^{-2}$, no dia 8 de julho de 1993. Segundo o mesmo autor, no dia 8, maior parte da partição de energia foi usada para aquecer a atmosfera pelo fluxo de calor sensível, ao invés da transpiração (LE), que é a maneira principal e usual de devolução de energia pelo bioma de floresta tropical.

O total integrado de energia em dias normais apresentou valores médios diários de 11,$6 ; 1,9$ e 6,3 MJ.m² dia $^{-1}$, para Rn, H e LE, respectivamente. Em dias de friagem, estes valores apresentaram uma redução para 7,0; 1,7 e 4,2 MJ.m

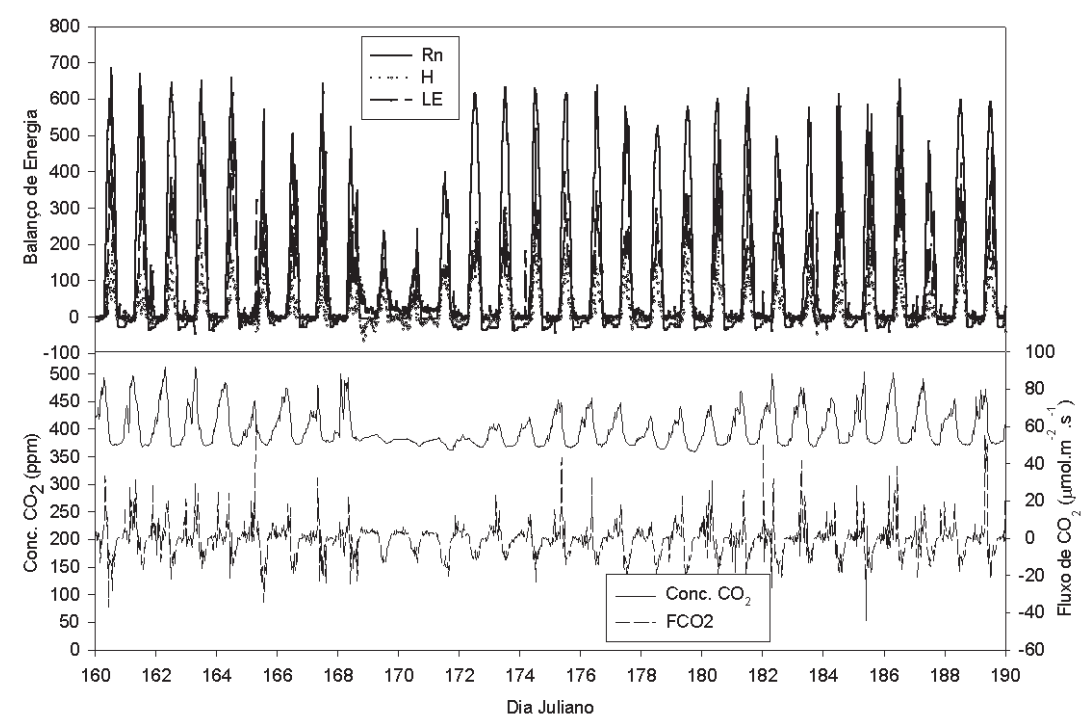

Figura 4 - Série Temporal dos fluxos de Energia e Carbono, durante o mês de Junho de 2001.

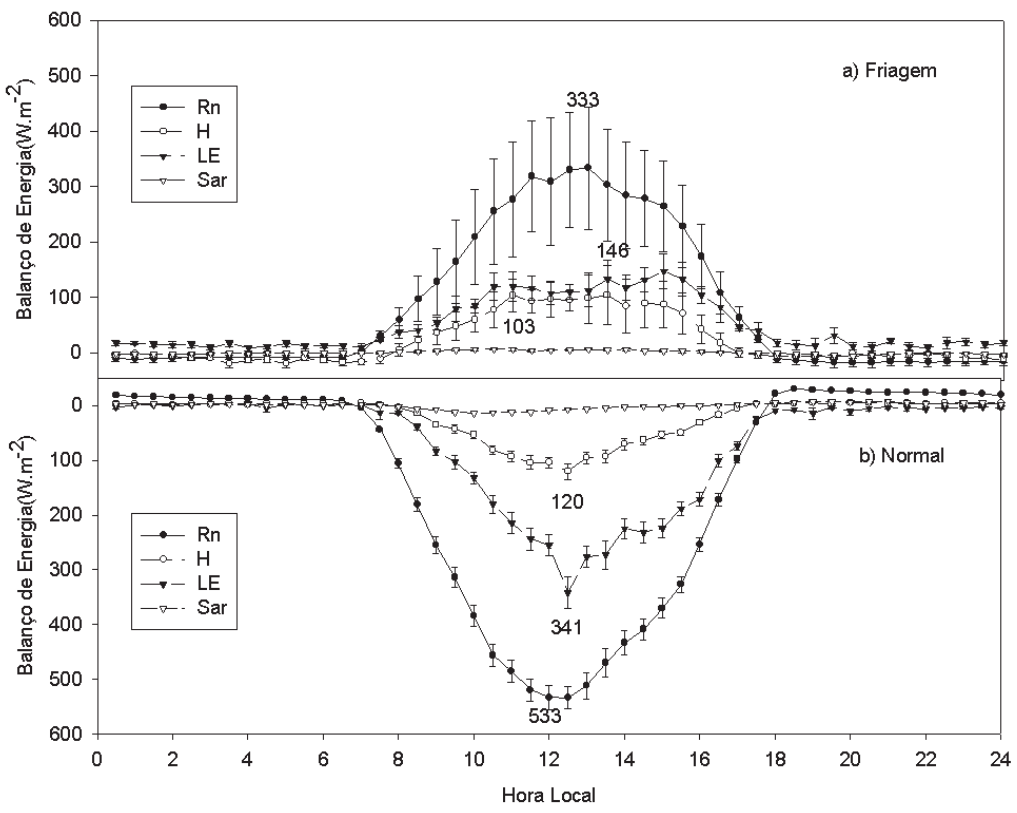

Figura 5 - Ciclo Diurno Médio do Balanço de Energia para dias de Friagem (a) e dias Normais (b)
${ }^{2} \mathrm{dia}^{-1}$, uma diferença de 39, 8 e $34 \%$, respectivamente (Tab. 2). Como citado anteriormente, observa-se que a maior redução foi encontrada na componente LE (34\%).

\section{Fluxo de carbono}

Nota-se na figura 4 que em dias de friagem a concentração de $\mathrm{CO}_{2}$ não aumenta durante a noite, permanecendo quase constante ao longo do dia, o que não acontece durante os dias normais, quando a concentração cresce durante a noite e logo em seguida começa a decrescer com o nascer do sol (Grace et al., 1996). Segundo Malhi et al. (1998), a natureza dos fluxos noturnos varia de acordo com as condições meteorológicas. Durante noites calmas, sob condições muito estáveis, a maior parte da respiração fica armazenada no dossel, sendo reabsorvida ou liberada no início da manhã (efluxo), enquanto que sob condições atmosféricas menos estáveis (devido à ação do vento forte) o fluxo de carbono é liberado intermitentemente durante a noite.

Percebe-se que devido à influência da friagem, os fluxos de $\mathrm{CO}_{2}$ são maiores durante a noite sem apresentar o pico máximo durante o início da manhã - efluxo (Fig. 6). As diferenças encontradas em tais condições são mostradas na Figura 6, a qual descreve o ciclo diurno do fluxo de $\mathrm{CO}_{2}$ medido pelo sistema "eddy covariance". Não se nota o efluxo durante o início da manhã em dias de friagem, o qual é uma resposta da liberação de carbono ocorrida durante a noite devido às condições de vento forte.

O pico máximo do fluxo de $\mathrm{CO}_{2}$ foi encontrado durante dias normais $(-14,7$ mmol $\left.\mathrm{m}^{-2} \mathrm{~s}^{-1}\right)$, maior que o valor encontrado durante dias de friagem (11,4 mmol $\mathrm{m}^{-2} \mathrm{~s}^{-1}$ ) (Fig. 6), provavelmente devido a maior intensidade de radiação solar incidente, sendo o vento, neste caso mais fraco e portanto um fator secundário. Nos resultados apresentados por Oliveira et al. (2001), sob condições normais (sem friagem), a radiação solar global não sofre muita variação, e o vento torna-se o principal controlador das trocas entre floresta e atmosfera.

De acordo com Oliveira et al. (2001), os fluxos de $\mathrm{CO}_{2}$ são maiores (mais negativo significa maior retirada de carbono pela floresta) em condições 
Tabela 2 - Componentes Médios do Balanço de Energia para dias de Friagem e Normais (MJ.dia'-1)

\begin{tabular}{|c|c|c|c|}
\hline & $\operatorname{Rn}($ MJ.dia-1) & $\mathrm{H}\left(\mathrm{MJ} . \mathrm{dia}^{-1}\right)$ & LE(MJ.dia $\left.{ }^{-1}\right)$ \\
\hline Dia normal & $11,61( \pm 0,3)$ & $1,88( \pm 0,1)$ & $6,34( \pm 0,2)$ \\
\hline $\begin{array}{l}\text { Dia de } \\
\text { friagem }\end{array}$ & $7,03( \pm 1,6)$ & $1,73( \pm 0,6)$ & $4,17( \pm 0,4)$ \\
\hline Diferença & $-4,58$ & $-0,15$ & $-2,17$ \\
\hline (\%) & -39 & -8 & -34 \\
\hline
\end{tabular}

normais com vento forte, do que sob condições de friagem. Os valores médios diários do fluxo de $\mathrm{CO}_{2}$ foram $-1,48 \mathrm{mmol}$

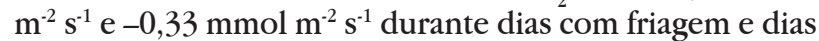
normais, respectivamente. Durante dias normais o fluxo médio diurno de $\mathrm{CO}$ foi de $-2,44 \mathrm{mmol} \mathrm{m}^{-2} \mathrm{~s}^{-1}$ aumentando, para $-5,78 \mathrm{mmol} \mathrm{m}^{-2} \mathrm{~s}^{-1} \mathrm{com}$ o efeito da friagem; enquanto que os fluxos médios noturnos foram $1,77 \mathrm{mmol} \mathrm{m}^{-2} \mathrm{~s}^{-1} \mathrm{e}$ $2,83 \mathrm{mmol} \mathrm{m}^{-2} \mathrm{~s}^{-1}$ durante dias normais e de friagem, respectivamente. (Tab. 3).

\section{CONCLUSÕES}

Este trabalho objetivou-se a estudar a influência da friagem nos fluxos de energia e carbono na Amazônia, onde se pôde concluir que, a friagem altera a partição de energia, causando uma redução de 7,03 MJ.dia ${ }^{-1}$ no saldo de radiação, 1,73 MJ.dia ${ }^{-1}$ no Fluxo de calor Sensível e 4,17 MJ.dia ${ }^{-1}$, no Fluxo de calor Latente. O fenômeno da friagem, também modifica o balanço de carbono na floresta, o qual apresentou fluxos médios diários de $1,48 \mathrm{mmolCO}_{2} \mathrm{~m}^{-2} \mathrm{~s}^{-1}$ durante o evento de friagem, e $0,33 \mathrm{mmolCO}_{2} \mathrm{~m}^{-2} \mathrm{~s}^{-1} \mathrm{em}$ dias normais, sendo esta intensificação no fluxo um resultado da maior eficiência nas trocas turbulentas em dias de friagem. Estas variações reduzem os valores dos elementos climáticos
Tabela 3 - Fluxo Médio de $\mathrm{CO}_{2}$ para dias de Friagem e dias Normais $\left(\mathrm{mmol} . \mathrm{m}^{-2} \cdot \mathrm{s}^{-1}\right)$

\begin{tabular}{lccc}
\hline \hline & \multicolumn{3}{c}{ Fluxos CO2 $\left(\mathrm{mmol} \mathrm{m}^{-2} \cdot \mathrm{s}^{-1}\right)$} \\
\cline { 2 - 4 } & $\begin{array}{c}\text { Média } \\
\text { diária }\end{array}$ & $\begin{array}{c}\text { Média: } \\
\text { dia }\end{array}$ & $\begin{array}{c}\text { Média: } \\
\text { noite }\end{array}$ \\
\hline Dia normal & $-0,33( \pm 0,3)$ & $-2,44( \pm 0,5)$ & $1,77( \pm 0,2)$ \\
Dia de friagem & $-1,48( \pm 0,4)$ & $-5,78( \pm 0,6)$ & $2,83( \pm 0,2)$ \\
\hline \hline
\end{tabular}

(principalmente dos valores de temperatura do ar), que podem impactar as atividades metabólicas das plantas. Atualmente discute-se muito o impacto do desmatamento da Amazônia no clima regional e global e um dos resultados é que sistema climático poderia ter mais eventos extremos (tal como a penetração mais freqüente de friagens). Este fato poderá provocar uma mudança do atual equilíbrio existente entre a floresta tropical e o clima. Os resultados apresentados podem ser úteis em estudos de modelagem atmosférica para futuros cenários da Amazônia.

\section{AGRADECIMENTOS}

Os autores agradecem ao Projeto LBA-EUSTACH pelo fornecimento dos dados referentes ao trabalho, bem como à equipe de Ji-Paraná/RO que trabalhou na coleta dos mesmos.

\section{BIBLIOGRAFIA CITADA}

Culf, A.D.; Esteves, J.L.; Marques Filho, A. de O.; Da Rocha, H.R. 1996. Radiation, temperature and humidity over forest and pasture in Amazônia. in: J. H. C. Gash, C. A. Nobre, J. M. Roberts, and R.L. Victoria (Eds). Amazonian Deforestation and Climate. John Wiley \& Sons, Chichester, England. p.175-192. Fisch, G. 1995. Camada Limite Amazônica: aspectos observacionais e de modelagem. Tese de Doutorado, Instituto Nacional de Pesquisas Espaciais, São José dos Campos, São Paulo. 125p.

Fisch, G.; Marengo, J. A.; Nobre, C.A. 1998. Uma Revisão Geral Sobre o Clima da Amazônia. Acta amazonica. 28(2): 101-126.

Fisch, G.; Tota, J.; Machado, L.A.T.; Silva Dias, M.A.F.; Lyra, R.F.da F.; Nobre, C.A.; Dolman, A.J.; Gash, J.H.C. 2004. The Convective Boundary Layer Over Pasture and Forest in Amazonia. Theoretical and Applied Climatology. (no prelo).

Fitzjarrald, D.R.; Moore, K. E. 1990. Mechanisms of Nocturnal Exchange Between the Rain Forest and Atmosphere. Journal of Geophysical Research, 95(D10): 16839-16850.

Figura 6 - Ciclo Diurno Médio do fluxo de $\mathrm{CO}_{2}$ durante dias Normais e de Friagem 


\section{ACTA AMAZONICA}

Grace, J.; Lloyde, J.; Mclintyre, J.; Miranda, A.C.; Meir, P.; Miranda, H.S. 1996. Carbon Dioxide flux over amazon rainforest in Rondônia. In: J. H. C. Gash, C. A. Nobre, J. M. Roberts, and R.L. Victoria (Eds). Amazonian Deforestation and Climate. John Wiley \& Sons, Chichester, England. p.307-318.

Kruijt, B.; Elbers, J.; Randow, C.V.; Araújo, A.; Culf, A.; Bink, N.J.; Oliveira, P.J.; Manzi, A.O.; Nobre, A.D.; Kabat, P.; Moors, E.J. 2004. The robustness in eddy correlation fluxes for Amazon rainforest conditions. LBA Special Issue. Ecological Application. (No prelo).

Malhi, Y., A.D. Nobre, J. Grace, B. Kruijt, M.G.P. Pereira, A. Culf and S. Scott. 1998. Carbon dioxide transfer over a central Amazonian rain forest. Journal of Geophysical Research, 103(24): 31593-31612.

Marengo, J.A.; Nobre, C.A.; Culf, A.D. 1997. Climatic Impacts of "Friagens" in forested and deforested areas of the Amazon Basin. Journal of Applied Meteorology, 36: 1553-1566.

Moncrieff, J.B. ; Massheder, J.M.; De Bruin, H.; Elbers, J.; Friborg, T.; Heusinkveld, B.; Kabat, P.; Scott, S.; Soeggard, H.; Verhoef, A. 1997. A system to measure surface fluxes of momentum, sensible heat, water vapour and carbon dioxide. Journal of Hydrology, 188-189: 589-611.

Oliveira A.P.; Fitzjarrald, D.R. 1994. The Amazon River Breeze and the Local Boundary Layer: II - Analysis and Modeling. Boundary Layer Meteorology, 67(1/7): 75-96.
Oliveira, P.j.; Fisch, G. 2001. Perfil Médio do Vento em Áreas de Floresta e Pastagem na Amazônia. Acta Amazonica, 31(4): 581-595.

Oliveira, P.J.; Kruijt, B.; Randow, C.V.; Manzi, A.O.; Waterloo, M. 2001. The Friagem Influence on $\mathrm{CO}_{2}$ Fluxes in a Forest in Rondonia. In: Global Change Open Science Conference: Challenges of a Changing Earth, Book of Abstracts, Amsterdam, Netherlands, p85.

Serra, A.; Rastibona, L. 1942. As massas de ar da América do Sul. Ministério da Agricultura, Serviço de Meteorologia. Rio de Janeiro, Rio de Janeiro, 32p.

Randow, C.V.; Manzi, A.O.; Kruijt, B.; Oliveira, P.J.; Zanchi, F.B.; Silva, R.L.; Hodnett, M.; Gash, J.; Elbers, J.; Waterloo, M.; Cardoso, F.L.; Kabat, P. 2004. The role of seasonal variations in exchange of energy and carbon over forest and pasture areas in Central West Amazonia. Theoretical and Applied Climatology. (No prelo).

RECEBIDO EM: 14/11/2002

ACEITO EM: 04/08/2004 
